\title{
MOBILE DEPLOYABLE LABORATORY - CHEMICAL MODULE
}

\author{
Constantin TOADER *, Gabriel EPURE*, Dănuţ MOŞTEANU**, \\ Cristiana EPURE*, Ovidiu IORGA*, Ilie FLORIN**
}

\author{
*Scientific Research Center for CBRN Defense and Ecology, Bucharest, Romania \\ **"Nicolae Bălcescu" Land Forces Academy, Sibiu, Romania \\ gabriel.epure@nbce.ro
}

\begin{abstract}
Changes in the layout of today's security medium, in which NATO and its member states evolve, in accordance to the new types of risks and threats, caused the reconsideration of the concept of security and modified priorities regarding civilian protection, territory and combatant forces.

The amplitude of the specific phenomenon associated with the proliferation of mass destruction weapons put the highest level of international political and military decisions into debate. In the given conditions, the field of CBRN defense becomes a strategic priority for the Alliance which decided (through the declaration of the general secretary of NATO at the summit in Prague) to adopt a set of engagements regarding the improvement of the defense capabilities against new threats through modernization and adaptation of specific structures. This paper presents the results of research conducted in the field of CBRN defense regarding the introduction of a "DEPLOYABLE Mobile CBRN Laboratory" within specific structures, product that will have the capability to be easily deployed in a combat theater, detect and identify (automatically or through instrumental analysis) $C B R N$ agents within a real or suspect CBRN contaminated medium and provide Intel that will help in real time decision processes that can evaluate the consequences of a CBRN event.
\end{abstract}

\section{Keywords: CBRN, mobile laboratory, surveillance, module}

\section{Introduction}

In the context of assuring strategic level measures, CBRN defense implies, according to national concepts, a distinct domain of great importance.

CBRN surveillance, integrated part of these measures, represents the ensemble of planned and executed actions destined to collect, analyze and evaluate data regarding the use of mass destruction weapons and/or the possibility that events with emissions other than an attack occur, with the purpose of evaluating the true level of CBRN threats, prevention, warning and assuring the protection of armed forces, civilians and the environment.

The CBRN surveillance and warning system (SSA CBRN), made within the Ministry of National Defense (MoND) represents the general organizational and functional platform through which CBRN surveillance mission fulfillments are assured. This product requires the capability to deploy CBRN detection and identification methods, so that the mission success is guaranteed trough implementing high end CBRN equipment.

\section{Destination}

The mobile deployable laboratory the chemical module is destined to perform missions, according to STANAG 4632 [1], acting in the following cases:

at the request of the Alliance with the approval of the home nation within the multinational CBRN defense 
Battalion;

trough national security system, in case of events of chemical nature that develop within the country's borders;

$\rightarrow$ at the request of international structures with the approval of the home nation, outside the country's borders, for specific detection and chemical data analysis - chemical module

The purpose of the Mobile deployable Laboratory - chemical module is to improve competences through expertise in sampling, preparation, analysis and identification of chemical warfare agents and toxic industrial chemicals, and also to support commanding structures in taking fast decisions in NATO operation areas.

The Mobile deployable Laboratory- chemical module will be capable of operating in any type of battlefield, in terrestrial operations, fulfilling missions that ranging from those concerning local security up to missions regarding collective defense.

\section{Product requirements}

The objectives, capabilities, structure, general and individual missions and other requirements for the deployable analytic laboratories were regulated through STANAG 4632 Ed.1„Deployable NBC Analytical Laboratory” and adopted nationally through the disposition of the State secretary for Weapons DA 11 from August 2012.

According to the requirements of the mentioned standard, the requirements presented in research contract, the diversity of situations in which the laboratory has to perform activities within the national territory and abroad, it is necessary that the endowment of the army with a deployable analysis laboratory, primarily the chemical module with high end performance equipment be of the highest priority.

The Mobile deployable Laboratorychemical module needs to: $\checkmark$ to offer commanding officers information so that they can take corresponding and informed decisions in case they encounter events of chemical nature within the country's borders or anywhere else where Romanian forces are deployed or joint actions under NATO are conducted;

$\rightarrow$ to offer the possibility of transportation by all possible means and anywhere in the zone of operations;

to be energetically independent;

to make laboratory analyses for acquiring information regarding chemical war agents or toxic industrial chemicals;

$\rightarrow$ to be equipped with materials that ensure at least 72 hours of continuous readiness;

to be fully compatible with the C3 system (Communications command and control) and equipped to fulfill the requirements of the „NATO HANDBOOK FOR SAMPLING AND IDENTIFICATION OF BIOLOCICAL; CHEMICAL AND RADIOLOGICAL AGENTS: AEP-66 (SIBCRA)" [2];

$\checkmark$ to be able to operate in diverse climates and geographical environments ($32 \div+49^{0} \mathrm{C}$ ), at a relative humidity of $90 \%$ (at $30^{\circ} \mathrm{C}$ );

to be equipped with devices that can be integrated in the CBRN Warning and surveillance system of the Romanian Army and to be compatible with NATO equipment.

\section{Container Configuration}

The integration variants will take into account the container configuration. It is comprised of three areas, as observed in figure nr.1:

1 - entrance, decontamination area and toilets;

2 - Sample reception compartment, preparation, analysis and identification

3 -technical compartment - the place where the electricity generator, helium and hydrogen pressurized containers, filtration and ventilation systems are situated etc.

Area 1 - the entrance, toilet and 
decontamination spaces will have a total length of $800 \mathrm{~mm}$ (value for the interior space) out of the total length of the container. While choosing this dimension, the size of the analytic equipment was taken into account and also the size of the chemical niche so that the introduction of these inside the container would be possible.

Area 2 -practically it is the area where all the equipment and systems that serve the mobile laboratory are placed. The length of this area differs for specific types of containers, for the ISO $1 \mathrm{C}$ container the available length for development will be of approximately $4000 \mathrm{~mm}$ and for the NATO $2 \mathrm{~S}$ container the available length will be $2000 \mathrm{~mm}$ [3].

Area 3 - the technical area, where the generator, gas containers and other systems will be placed has an available length of $1,000 \mathrm{~mm}$ as it is seen in the schematics of the equipment and the positioning of these in the available space.

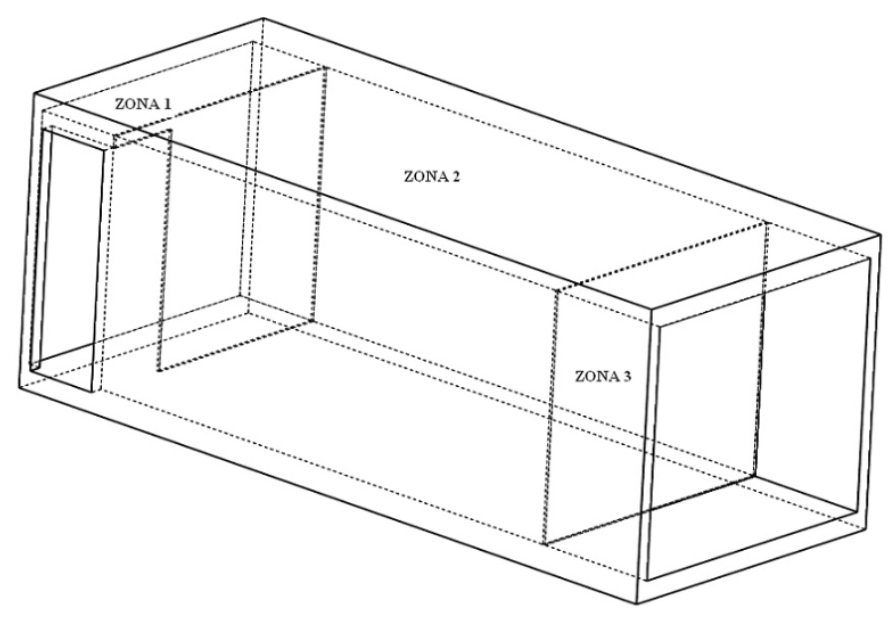

Figure 1. Container configuration

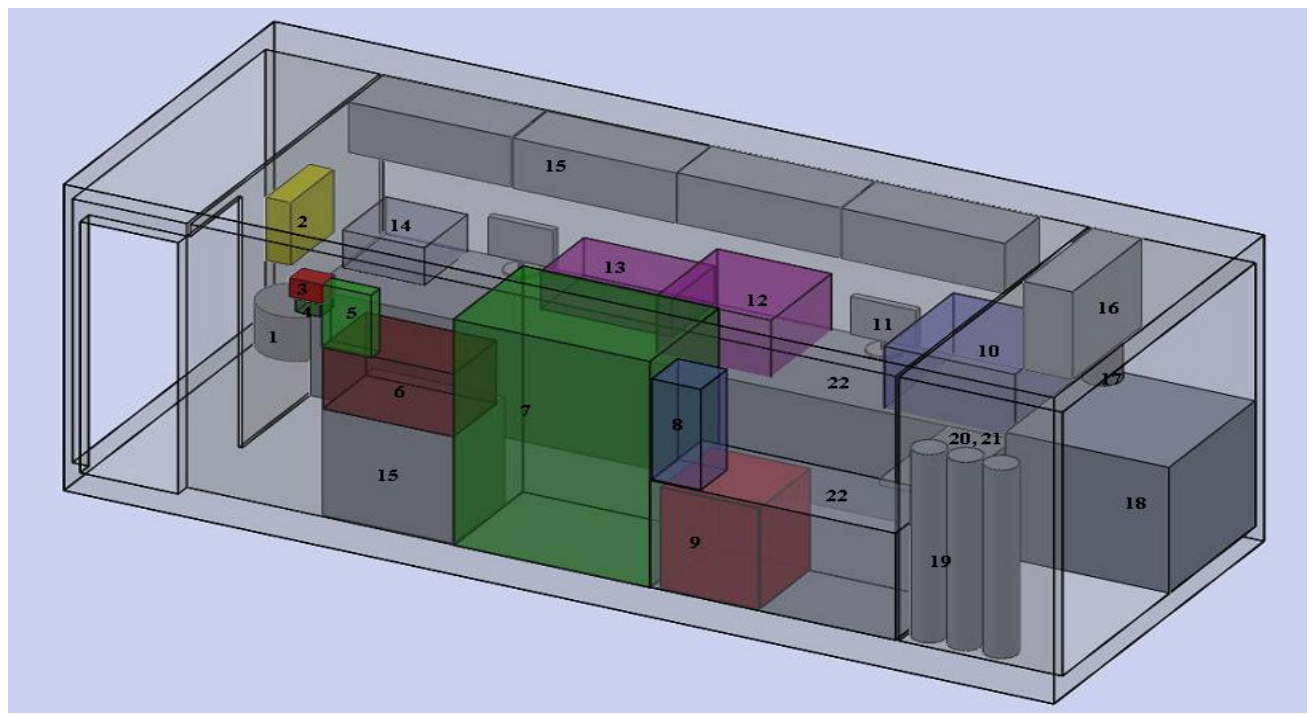

Figure 2.Placement of analysis and preparation equipments in the ISO-1C container 


\section{Optimal Integration Variant}

During the development of the project, simulations were conducted for the placement of equipment in two other types of container, this article presenting the optimal result. (figure 2).

In figure 2 the placement of equipment in the container type ISO 1C.

Legend:

1. toilet, decontamination area;

2. central command panel: generator switch, filtration and ventilation, climate control, electricity etc.;

3. radiological detection system;

4. chemical detection system;

5. stationary chemical agent detection system;

6. gloovebox niche for receiving contaminated samples and decontamination system; preparation;

7. chemical niche, sample system;

8. automatic sample extraction

9. refrigerator for the received or stored samples;

10. analysis system GC-MS;

11. displays;

12. analyses system GC - FPD;

13. FTIR system;

14. XRAY system;

15. suspended storage locations for books materials etc.;

16. air conditioning splitter;

17. filtration and ventilation systems

18. electricity generator;

19. special gas cylinders;

20. radio station;

21. data unit;

22. lab furniture.

Advantages:

- within this type of container with a length of $4000 \mathrm{~mm}$ systems can be integrated; this type of container is used by other NATO members which have a mobile deployable laboratory as well;

$\checkmark$ there is optimal space for optimal activity development and flux, this being correlated with the careful placement of the devices and systems;

Disadvantages:

- no disadvantages compared to the other studied models where found

$\checkmark$ there is a small insufficiency in the maneuverability of the container due to its increased mass

The integration of equipment and systems on this type of container is the ideal variant because all equipment and systems proposed in the concept study can be included on the ISO 1C container.

After choosing the optimal variant, the technical execution project was elaborated so that the container would be fitted according to the requirements of the technical specifications.

Further plans for this project are the integration of the filtration and ventilation system, climate control and adding the analytic systems to the container.

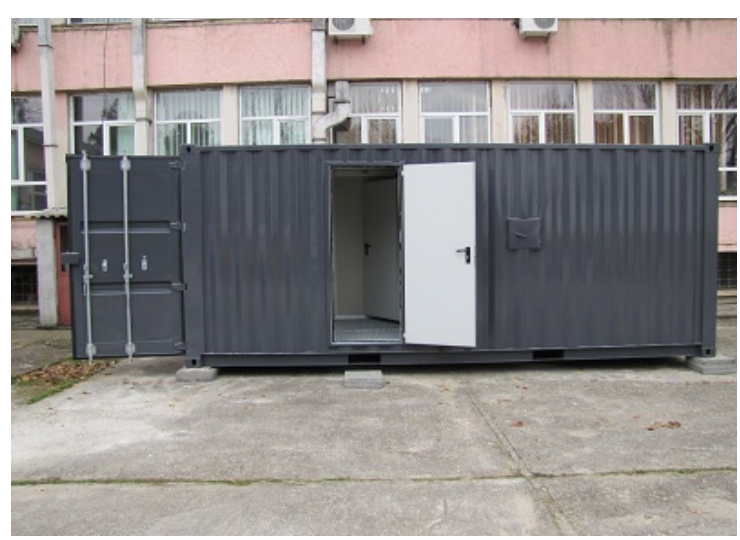

Figure 3. Container ISO1C front view

\section{References}

[1] STANAG 4632 Ed.1 - „Deployable NBC Analytical Laboratory”.

[2] AEP-66 (SIBCRA) - NATO handbook for sampling and identification of biological chemical and radiological agents.

[3] European Commission Customs 2002: Good practice guide, Container specifications. 